\title{
Da Fabricação Digital para o Design: propriedades emergentes e implicações
}

\author{
From Digital Fabrication to Design: emergent properties and implications
}

MINEIRO, Érico; Dr.; Universidade Federal de Minas Gerais

ericomineiro@ufmg.br

MAGALHÃES, Claudio; Dr.; Pontifícia Universidade Católica do Rio de Janeiro

claudio-design@puc-rio.br

\section{Resumo}

Práticas de design são, com frequência, influenciadas por particularidades de processos de fabricação. Ocorre que a crescente disponibilidade de tecnologias digitais de produção tem transformado a própria ideia de fábrica. Neste contexto desponta o entendimento de que as práticas e a educação em design devem considerar as mudanças no cenário tecnológico. Em especial as implicações para espaços de possibilidades delineados nas interfaces entre projeto e produção. Na medida em que tecnologias de fabricação digital se difundem e são inseridas nas práticas de projeto e desenvolvimento, emergem propriedades que até então não eram - ou não poderiam ser - observadas. A fim de subsidiar reflexões sobre estas transformações, este trabalho tem como objetivo caracterizar sistemas de fabricação digital e evidenciar propriedades emergentes. Para tanto, sistemas de fabricação digital são caracterizados em sua diversidade, propriedades emergentes são identificadas e implicações para o design são discutidas.

Palavras Chave: fabricação digital; design; tecnologias de produção.

\begin{abstract}
Design practices are often influenced by particularities of manufacturing processes. It turns out that the increasing availability of digital production technologies has transformed the very idea of the factory. In this context emerges the understanding that practices and education in design must consider the changes in the technological scenario. In particular the implications for spaces of possibilities delineated in the interfaces between design and production. As digital manufacturing technologies spread and are embedded in design and development practices, properties that previously were not - or could not be - observed, emerge. In order to support reflections on these transformations, this work aims to characterize and highlight emerging properties of digital manufacturing systems. To this end, digital manufacturing systems are characterized in their diversity, emerging properties are identified and implications for design are discussed.
\end{abstract}

Keywords: digital fabrication; design; manufacturing technologies. 


\section{Introdução}

Desde suas primeiras manifestações formais, o ensino e as práticas profissionais de design mantêm espaço para conhecimento teórico e experimentação com materiais e processos de produção. Boa parte da organização pedagógica da Bauhaus girou em torno do estudo de materiais e das práticas de oficina (BÜRDEK, 2006, p. 29-31).

O design surge como atividade profissional vinculado à industrialização e à produção em massa, neste contexto o ensino do design mais servia à indústria do que conseguia influenciá-la (BUCHANAN, 1998, p. 64).

Ao lado do desenvolvimento da industrialização, observa-se uma ênfase do design industrial na configuração prático-funcional, a fim de atender aos interesses da produção industrial (LÖBACH, 2001, p. 89-90). Os ideais funcionalistas de racionalização podem ser notados nos princípios do 'bom design' de Dieter Rams, que refletem a eficiência produtiva enquanto valor e terminam por incorporá-la como um dos critérios de qualidade para o design.

A atenção dedicada à interface entre projeto e produção atravessou décadas e ganhou forma e sofisticação metodológica nas abordagens de DfM (Design for Manufacturing) e em outros instrumentos relacionados à produtividade e qualidade na produção, como o QFD (Quality Function Deployment) (CLARK e WHEELWRIGHT, 1993).

Ao longo do tempo a complexidade observada nas práticas de design, em suas saídas e em seu entorno, é notável. Um 'bom design' deve ser bem-sucedido em múltiplas dimensões, que ao mesmo tempo incluem e transpassam as relações entre projeto e produção (HERTENSTEIN, PLATT e VERYZER, 2013).

Estas múltiplas dimensões ou domínios, são objeto de conjectura e apreciação (julgamento) nos processos mais essenciais das práticas de design (SCHÖN, 1983, p. 76-104).

Em meio a esta complexidade, a viabilidade técnica e econômica de proposições de design tem suas questões mais fundamentais na interface entre projeto e produção.

Nos cursos de design de produto os acertos muitas vezes difíceis entre boas ideias de design e as realidades do chão de fábrica são um tema recorrente de aprendizagem, um bom designer deve se assegurar que suas propostas de design sejam realmente factíveis, realizáveis pela produção (LIPSON e KURMAN, 2013, p. 32, 175).

A partir destas considerações pode-se afirmar sem incorrer em grande risco que, não raro, nas práticas de design a forma segue a fabricação ou, em outras palavras, as tecnologias de fabricação delineiam o espaço de possibilidades para o design daquilo que pode ser realizado.

Ocorre que a própria ideia de 'fábrica' está em transformação, em especial por decorrência da crescente disponibilidade de tecnologias digitais de produção (ANDERSON, 2012, p. 16), ou seja, pela difusão das tecnologias de fabricação digital, dos processos de produção de artefatos físicos a partir de dados digitais.

Tipicamente estes processos envolvem projetar algo em um sistema CAD, definir como será sua produção em um sistema CAM e então executar o código criado em uma máquina de modo que ela produza objetos tangíveis reais (FORD, 2016, p.2).

Cabe observar que, embora as primeiras máquinas de usinagem controlada por 
computador (CNC) tenham sido criadas ainda em meados do século XX e as primeiras tecnologias de impressão 3D tenham sido inventadas há cerca de 30 anos, a difusão das tecnologias de fabricação digital se iniciou de fato apenas nos primeiros anos do século XXI.

Artefatos impressos em 3D com qualidade suficiente para que fossem entendidos como produtos surgiram apenas no início dos anos 2000 (ANTONELLI, 2011) e, a título de exemplo, a primeira impressora 3D de mesa ${ }^{1}$ foi lançada em 2007 (REPRAP, 2011).

Assim sendo, tecnologias de fabricação digital, como impressoras 3D e máquinas de usinagem CNC, não são exatamente novas tecnologias. O que realmente há de novo é a difusão e a crescente disponibilidade de acesso a estas tecnologias, acompanhadas das melhorias incorporadas desde que foram criadas.

Paralelamente surgiram ainda espaços compartilhados dedicados, como os Fab Labs e sistemas geograficamente distribuídos de fabricação digital.

Como Gershenfeld constata:

\begin{abstract}
Uma nova revolução digital está em curso, desta vez na fabricação. Ela se baseia nas mesas ideias que levaram anteriormente à digitalização da computação e da comunicação, mas agora o que está sendo programado é o mundo físico, mais do que o virtual. A fabricação digital permitirá indivíduos projetarem e produzirem objetos tangíveis sob demanda, quando e onde eles precisarem. O acesso pulverizado destas tecnologias desafiará modelos tradicionais de negócios, de apoio e educacionais (GERSHENFELD, 2012, p.43).
\end{abstract}

A previsão feita por Schrage parece ter se concretizado: a redução da complexidade e dos custos dos processos de fabricação digital estão transformando a prototipagem física, de maneira análoga ao modo como a publicação desktop ampliou possibilidades e oportunidades para o design gráfico (2000, p. 83).

Retomando o entendimento de que o design industrial, como conhecido, é estreitamente relacionado aos sistemas técnicos industriais, então uma transformação tecnológica deveria ser acompanhada por uma transformação do design, com implicações sobre a educação e sobre as práticas profissionais (MAGALHÃES, 2014, p. 155).

Nesse sentido, este trabalho tem como objetivo evidenciar propriedades emergentes da difusão de tecnologias de fabricação digital, de modo que se possa subsidiar reflexões e discutir implicações para o design. Ocorre que, na medida em que estas tecnologias são difundidas e inseridas nas práticas de projeto e desenvolvimento, emergem novas propriedades que até então não eram - ou não poderiam ser - observadas.

Assim, entende-se ser necessário que, ainda antes de se pensar o 'design para a fabricação digital', sejam identificadas as propriedades que emergem destes sistemas tecnológicos quando colocados em funcionamento, ou, inseridos em contextos de uso, para que se possa refletir sobre suas implicações para as práticas profissionais e para o ensino.

Um caminho no sentido da fabricação digital para o design.

\footnotetext{
1 Equipamentos desktop, de mesa, ou de bancada, assim considerados em função da redução dimensional, tipicamente acompanhada de redução da complexidade operacional e de custos de aquisição.
} 


\section{Da Fabricação Digital para o Design}

No desenvolvimento deste trabalho as tecnologias mais conhecidas de fabricação digital são brevemente apresentadas (seção 2.1), propriedades emergentes já conhecidas de tecnologias de impressão 3D são evidenciadas (seção 2.2), assim como algumas das principais formas de associação entre estas tecnologias e tecnologias tradicionais de produção (seção 2.3), em seguida, tipos de sistemas formados por tecnologias de fabricação digital são brevemente caracterizados (seção 2.4), implicações da adoção de tecnologias de fabricação digital relacionadas à produção de pequenos lotes e customização em massa são identificadas (seção 2.5), assim como limitações conhecidas destas tecnologias (seção 2.6) e avanços tecnológicos esperados para o futuro (seção 2.7).

\subsection{As Tecnologias de Fabricação Digital}

As impressoras 3D, presumivelmente, representam as perspectivas mais expressivas de efeitos decorrentes da fabricação digital, contudo, outras tecnologias também são parte importante neste corpo de avanços técnicos.

Para Chris Anderson, por exemplo, as máquinas de corte a laser são "o verdadeiro cavalo de carga do movimento maker" (2012, p. 109).

Os primeiros Fab Labs, espaços compartilhados de fabricação digital, não contavam com impressoras 3D (GERSHENFELD, 2005, p. 12). Para ser reconhecido como um Fab Lab, um laboratório deve compreender um conjunto de tecnologias que contenha, ao menos, uma máquina de corte a laser, uma fresadora CNC, um plotter de recorte e uma bancada para prototipagem de circuitos eletrônicos (FAB FOUNDATION, 2015).

Entre as tecnologias mais difundidas de fabricação digital estão as fresadoras CNC, as máquinas de corte a laser e as impressoras 3D.

O que estas máquinas têm em comum é a capacidade de interpretar códigos gerados por sistemas CAD-CAM e executar com precisão transformações em materiais físicos a partir destes códigos.

Em particular, as tecnologias de impressão 3D mais comuns podem ser descritas como sistemas de produção por adição de material e deposição de camadas, uma vez que as camadas de materiais são criadas de modo subsequente, umas sobre as outras, na construção dos artefatos físicos, em alguns dos casos por deposição de material, em outros pela sinterização ou fotopolimerização de material em pó ou em estado líquido.

Os dois outros processos operam pela retirada de material, enquanto as máquinas a laser executam cortes rápidos em um processo simplificado, fresadoras CNC não apenas são capazes de executar cortes em chapas, como também de usinar modelos sólidos tridimensionais em processos mais elaborados que envolvem trocas de ferramentas durante a produção, configurações de parâmetros como velocidades e avanços das ferramentas sobre os materiais e em alguns casos mais de três eixos de operação.

Tipicamente, estas tecnologias vêm sendo utilizadas para a construção de modelos rápidos e de baixa fidelidade (quick-and-dirty prototyping). A estas práticas, assim como às tecnologias envolvidas na produção destes modelos, é atribuída a denominação de 'prototipagem rápida' (GIBSON, ROSEN e STUCKER, 2010; VOLPATO, 2007). 
Embora estas tecnologias - particularmente a impressão 3D - tenham sido associadas à prototipagem de baixa fidelidade e à produção amadora de pequenos objetos, elas também têm sido utilizadas na fabricação de produtos e componentes finais (HOPKINSON, HAGUE e DICKENS, 2006).

Melhorias na qualidade das máquinas de prototipagem rápida crescentemente aproximam as saídas destes processos da qualidade de produtos. De fato, muitas peças já são produzidas diretamente nestas máquinas.

Avanços significativos de precisão e de qualidade de materiais têm catapultado estas tecnologias para testes, ferramental, produção e outros âmbitos que estão além da definição de protótipo (GIBSON, ROSEN e STUCKER, 2010, p. 8).

Por estes motivos há um consenso crescente em relação à adoção do termo 'manufatura aditiva' em substituição ao termo 'prototipagem rápida' para descrever as tecnologias de impressão 3D.

Assim, estas tecnologias deixam de ser máquinas que servem somente à construção de modelos e protótipos para serem reconhecidas também como equipamentos capazes de fabricar produtos e partes de produtos, equipamentos de fabricação digital.

\subsection{Propriedades Emergentes conhecidas em Sistemas de Impressão 3D}

Uma revisão da literatura indica que algumas propriedades que despontam no uso destes sistemas técnicos são conhecidas, em especial, para as tecnologias de impressão 3D. O Quadro 1 apresenta uma síntese desta revisão.

Quadro 1 - Propriedades emergentes conhecidas de sistemas de impressão 3D

\begin{tabular}{ll}
\hline Propriedade & Descrição \\
\hline Complexidade formal & Tecnologias de impressão 3D permitem a construção de formas \\
& complexas, dispensando ângulos de saída de moldes, permitindo assim \\
& ângulos negativos, cavidades e câmaras. O volume das peças construídas \\
determina custos de produção, que são pouco ou nada afetados pela & complexidade formal. \\
\hline
\end{tabular}

Variedade interartefatos

A variedade de artefatos produzidos, inclusive em um mesmo ciclo de produção, não impacta custos e tempo de produção.

Tempo de ajustes reduzido

Não há necessidade de ferramental ou de ajustes e configurações iniciais complexos, a interação do operador com o processo de produção se dá em poucos passos e muitas vezes há pouco ou nenhum trabalho pósprodução.

Variedade multimaterial

Algumas tecnologias de impressão $3 D$ permitem a construção multimaterial em um mesmo ciclo de produção, com isso propriedades materiais distintas, óticas, mecânicas ou elétricas podem ser empregadas na construção de um mesmo sólido.

Fonte: sintetizado de GERSHENFELD, 2005; HOPKINSON, HAGUE e DICKENS, 2006; GIBSON, ROSEN e STUCKER, 2010; LIPSON e KURMAN, 2013. 


\subsection{Associações entre Tecnologias de Fabricação Digital e Processos Produtivos Tradicionais}

A integração entre tecnologias de fabricação digital e processos produtivos tradicionais pode se dar de diferentes maneiras (Quadro 2).

A forma mais evidente de associação é a adoção de tecnologias de fabricação digital para prototipagem nas práticas de design, seguida pela fabricação por processos produtivos convencionais.

Partes e componentes já fabricados por processos maduros podem ser inseridos durante a fabricação digital. Assim, pequenos componentes capazes de desempenho superior aos similares produzidos por fabricação digital podem ser utilizados como insertos. Partes com funções mecânicas (como rolamentos, roscas e molas) e componentes com outras funções (como condutores elétricos, componentes e circuitos eletrônicos) podem ser inseridos durante a fabricação digital ou montados após a construção.

Tecnologias de fabricação digital podem servir também à construção de matrizes que venham a ser inseridos em processos produtivos maduros, ou ainda, na construção de moldesprotótipo, que podem ser usados com ferramentas porta-molde para a produção em equipamentos tradicionais de fabricação (VOLPATO, 2007, p. 163-65).

A complexidade formal permitida pelas tecnologias de fabricação digital torna possível a construção de peças consolidadas, ou seja, peças que em processos de fabricação tradicionais precisariam ser um conjunto montado de partes produzidas separadamente. Deste modo peças consolidadas produzidas por fabricação digital podem ser montadas com outras peças, menos elaboradas, mas com melhor desempenho ou custo, produzidas por tecnologias convencionais.

Quadro 2 - Formas conhecidas de associação entre fabricação digital e fabricação convencional

\begin{tabular}{lll}
\hline & Papel da fabricação digital & Papel da fabricação convencional \\
\hline (A) & Prototipagem & Fabricação do produto \\
\hline (B) & Fabricação do produto & $\begin{array}{l}\text { Componentes pré-fabricados } \\
\text { (inseridos durante a fabricação digital ou montados após) }\end{array}$ \\
\hline (C) & Fabricação de matrizes ou moldes-protótipo & Fabricação do produto \\
\hline (D) & $\begin{array}{l}\text { Fabricação de partes consolidadas de } \\
\text { geometria complexa }\end{array}$ & $\begin{array}{l}\text { Fabricação de componentes (montados junto às partes } \\
\text { consolidadas produzidas por fabricação digital) }\end{array}$ \\
\hline
\end{tabular}

Fonte: elaborado pelos autores.

Além destas formas de associação, softwares, sensores, controles e acionadores digitais têm sido inseridos em processos convencionais, constituindo sistemas tecnológicos elaborados, em um processo conhecido como 'transformação digital', que permite a criação de sistemas virtuais e físicos interconectados que podem alcançar níveis altos de flexibilidade de produção.

Esta 'transformação digital' seria o principal processo de transição de uma indústria convencional para a indústria 4.0. 


\subsection{Sistemas Dedicados, Ampliados e Distribuídos de Fabricação Digital}

Uma alternativa à transição analógico-digital citada na seção anterior é a constituição de sistemas formados exclusivamente por associações entre tecnologias digitais. Outras propriedades emergem a partir destas articulações.

A obrigatoriedade de um sistema tecnológico mínimo, para que um laboratório seja reconhecido como um Fab $L a b$, por exemplo, serve não apenas para assegurar possibilidades múltiplas de combinações entre tecnologias de fabricação digital, mas, mais do que isso, facilita colaborações - inclusive internacionais - entre laboratórios (FAB FOUNDATION, 2015).

Compartilhar, replicar, testar e modificar artefatos sem limitações geográficas são possibilidades que surgem a partir da implementação de sistemas dedicados de fabricação digital.

Em outra perspectiva, três outros grupos de tecnologias se destacam na formação de sistemas ampliados de fabricação digital: (a) tecnologias de captura da forma tridimensional; (b) programas de modelagem tridimensional digital; e (c) dispositivos óticos e hápticos de captura de movimentos para interações humano-computador.

As tecnologias de captura da forma tridimensional, variam de softwares que processam fotografias digitais em nuvem aos equipamentos de diagnóstico por imagem não invasivo (tomografia computadorizada, ultrassonografia e ressonância magnética), passando por scanners tridimensionais de mesa amadores, e outros, de uso industrial para controle dimensional de qualidade. A integração destas tecnologias em sistemas ampliados de fabricação digital tem permitido resultados surpreendentes em áreas diversas, como a arqueologia, a paleontologia, a medicina, o design e as artes (LOPES, et al., 2013).

Já os programas de modelagem digital tridimensional, embora sejam tecnologias essenciais nestes sistemas, muitas vezes representam mais um gargalo para o desenvolvimento e a produção do que efetivamente servem como sistemas de apoio ao design.

Muitos destes programas não se mostram adequados à gama de possibilidades abertas pelas tecnologias de fabricação digital, em especial as de impressão 3D, que propiciam muito mais possibilidades construtivas do que aquelas para as quais estes ambientes de modelagem virtual foram criados.

O relacionamento [entre impressão $3 \mathrm{D}$ e programas de modelagem tridimensional] tem sido unilateral. A impressão 3D se desenvolveu sobre programas de modelagem. Contudo os programas de modelagem não se desenvolveram em função da impressão 3D. (LIPSON e KURMAN, 2013, p. 91)

Os programas CAD baseados em modelagem de sólidos apresentam várias limitações quando inseridos em sistemas de fabricação digital, em especial as dificuldades em permitir construções geometricamente complexas, com centenas ou milhares de variações formais (GIBSON, ROSEN e STUCKER, 2010, p. 301).

Finalmente, estes sistemas podem ser ampliados com a inserção de periféricos de interação humano-computador utilizados na modelagem tridimensional digital, entre eles os dispositivos hápticos, que propiciam uma interface tátil rica para a criação de modelos tridimensionais; de outro lado, periféricos de captura tridimensional de movimento têm sido 
utilizados em experimentos de interação humano-computador e potencialmente podem vir a integrar estes sistemas.

Todas estas tecnologias têm o potencial de estender as potencialidades de utilização e de fazer emergir propriedades ainda desconhecidas em sistemas de fabricação digital ampliados.

Em uma terceira perspectiva, algumas das mudanças mais substanciais introduzidas pela fabricação digital decorrem da facilidade de transferência de arquivos digitais, que abre possibilidades até então impensadas para produção em sistemas distribuídos de fabricação digital.

Uma das implicações dos desenvolvimentos recentes da fabricação digital é o questionamento das razões fundamentais para a centralização das atividades produtivas e de desenvolvimento de produtos, que passam a poder ser distribuídas independentemente da localização espacial-geográfica (GIBSON, ROSEN e STUCKER, 2010, p. 437).

Sistemas de fabricação digital virtualmente interconectados - conhecidos como hubs de fabricação digital - têm se apresentado como uma alternativa para a produção em baixa escala e de objetos únicos.

Plataformas digitais criam redes de equipamentos de pessoas físicas com capacidade ociosa, fazendo com que esta capacidade seja acessível como um serviço para terceiros ${ }^{2}$.

A descentralização da produção aliada às tecnologias digitais abre ainda a possibilidade de produção no ponto da demanda, eliminando a necessidade de distribuição física de produtos, eliminando estoques e fazendo possível um just-in-time verdadeiro (HOPKINSON, HAGUE e DICKENS, 2006, p. 162-65).

Outros modelos de produção distribuída são oferecidos por empresas que prestam serviços de produção digital (bureaus de fabricação digital). Em alguns casos os serviços compreendem fabricação e a comercialização online dos projetos/produtos com alcance internacional ${ }^{3}$.

Alguns destes sistemas distribuídos de fabricação digital oferecem acesso a equipamentos de fabricação digital de alto nível, até então inacessíveis para usos e aplicações que não estivessem compreendidas nos interesses das grandes indústrias.

Neste cenário não é difícil imaginar que a diversidade de sistemas de fabricação digital aqui retratada favorecerá a criação de artefatos físicos inovadores, se não pela ampliação da gama de artefatos viáveis, presumivelmente pela multiplicidade de arranjos de fabricação possíveis decorrentes da crescente disponibilidade destes sistemas tecnológicos.

A inovação floresceu rapidamente na indústria de software porque o custo de entrada é menor no mundo virtual do que no mundo físico, de maneira análoga, os hubs de fabricação digital [assim como os demais sistemas de fabricação digital, ampliados e distribuídos] deverão impulsionar a inovação de produtos físicos (LIPSON e KURMAN, 2013, p. 47).

\footnotetext{
2 A título de exemplo, a 3D Hubs, fundada em 2013 integra mais de 25.000 equipamentos de fabricação digital entre impressoras 3D e fresadoras CNC, distribuídas entre mais de 150 países. <www.3dhubs.com/trends>. Acesso em mar. 2018.

3 O modelo de produção distribuída de uma destas empresas consultada durante esta pesquisa oferece serviços de impressão 3D e comercialização de modelos impressos para mais de cem países.
} 


\title{
2.5 Pequenos Lotes e Customização em Massa na Fabricação Digital
}

Investimentos e custos iniciais altos - inclusive custos ocultos - associados ao desenvolvimento de produtos para a indústria, contexto no qual a economia de escala é o fator que justifica o investimento, fazem com que a ideia de 'produção industrial em pequenos lotes' seja um paradoxo (LIPSON e KURMAN, 2013, p. 25-27).

De outro lado, as tecnologias de fabricação digital combinam precisão digital com a replicabilidade do chão de fábrica e liberdade de design comparável à do artesão (Ibid.).

A dispensa de moldes na fabricação digital direta implica na redução de boa parte dos custos e do tempo que antecedem o primeiro ciclo de produção. Estes fatores, associados à redução dos recursos humanos necessários para fabricação, reduzem riscos de lançamento de novos produtos e tornam possível a produção rápida e econômica em lotes pequenos e médios (HOPKINSON, HAGUE e DICKENS, 2006, p. 171).

As tecnologias de fabricação digital alteram a própria noção de 'lote'. Uma vez que a variabilidade em si não gera impactos sobre a produção, a customização em massa passa a ser uma possibilidade real ${ }^{4}$ e a ideia de lote passa a ser definida mais pelo conjunto qualitativo de produtos agregados em cada ciclo de produção, do que apenas pela quantidade de produtos produzidos.

Assim, o lote pode ser determinado pelo momento no tempo em que foi feito um conjunto de demandas, por características dos produtos que serão fabricados, ou pelo agrupamento de demandas por localização geográfica, por exemplo.

Como afirmou Antonelli (2011), cada objeto impresso em 3D pode ser compreendido, ao mesmo tempo, como um protótipo e um elemento de uma série diversificada de produtos.

\subsection{Limitações das Tecnologias e Sistemas de Fabricação Digital}

Mesmo impactantes e promissoras, as tecnologias fabricação digital também carregam suas limitações.

Como Gershenfeld observou a respeito das impressoras 3D, em muitos casos, o tempo do ciclo de produção é longo e os custos dos equipamentos são altos.

\begin{abstract}
Apesar do apelo óbvio de serem capaz de imprimir quase qualquer coisa, atualmente as impressoras 3D tem um número de limitações significativas. Elas são precificadas mais como mainframes do que como impressoras, e o que elas fazem poderia ser chamado mais precisamente de prototipagem não-tão-rápida. O tempo de construção de uma peça complexa pode variar de horas até dias. (GERSHENFELD, 2005, p. 100)
\end{abstract}

Companhias fabricantes de tecnologias de impressão 3D atuam em mercados bastante distintos e acabam por determinar duas grandes classes de equipamentos: uma direcionada para a fabricação digital de alto nível industrial, e outra, de tecnologias de mesa voltadas para o consumidor comum e para educação; duas classes de equipamentos com desempenho e custos envolvidos muito distantes.

Em geral o que se verifica é que as impressoras 3D desktop de baixo custo ainda não alcançam níveis muito bons de qualidade estrutural ou de acabamento do artefato produzido.

\footnotetext{
${ }^{4}$ Gibson, Rosen e Stucker (2010, p. 371) citam um caso real em que se produz por estereolitografia até 4000 invólucros de aparelhos auditivos customizados individualmente em apenas um ciclo de produção.
} 
As tecnologias desktop capazes de imprimir objetos de alta resolução são caras, assim como seus insumos e, ao mesmo tempo, apresentam limitações de área útil com implicações para as dimensões do artefato (como as tecnologias de estereolitografia).

Os materiais disponíveis para impressão 3D muitas vezes não têm a mesma qualidade dos materiais similares empregados na indústria tradicional (VOLPATO, 2007, p. 11).

Problemas com falhas por falta ou excesso de material, ou por variações não desejáveis dimensionais e de propriedades físicas são frequentes (GIBSON, ROSEN e STUCKER, 2010, p.10).

De outro lado, os sistemas distribuídos de produção apresentam outros tipos de limitações.

Ambientes e práticas de produção industrial são muito mais rigorosos do que aqueles de prototipagem, inclusive em termos de set-up (ajustes iniciais), calibração, controle de qualidade, certificação e treinamento de pessoal (GIBSON, ROSEN e STUCKER, 2010, p.372).

Raramente este conjunto de práticas pode ser encontrado em sistemas distribuídos de fabricação digital. Inconsistências entre procedimentos (como ajustes de máquinas) e propriedades materiais esperados e os reais, colocam em risco qualidade e viabilidade econômica da fabricação digital de produtos.

\subsection{Avanços Esperados}

Na convergência entre os mundos digital e físico, proporcionada pelas tecnologias de fabricação digital, o controle sobre a produção deve ser conquistado progressivamente: inicialmente seria preciso conquistar o controle sobre a forma; em seguida, sobre a composição da matéria; e, em um terceiro momento, o controle sobre comportamento dos artefatos, por meio da "habilidade de programar materiais para funcionar de modos desejados" (LIPSON e KURMAN, 2013, p. 265).

Nestes três níveis de domínio, os dois últimos ainda dependem de desenvolvimento tecnológico.

Algumas das tecnologias de impressão 3D depositam materiais ponto a ponto, de modo discreto, não necessariamente contínuo. Ao operar com mais de um material em pontas de deposição múltiplas, estas tecnologias são potencialmente capazes de construir 'materiais funcionalmente graduados' (FGMs, functionally graded materials) nos quais misturas de materiais em diferentes proporções permitiriam composições variadas, com propriedades físicas também variadas em uma mesma construção, ou seja, seria possível a variação espacial controlada de propriedades materiais: mecânicas, elétricas, térmicas e óticas (OXMAN, KEATING e TSAI, 2012).

Aparentemente a alternativa mais consistente para tornar possível a distribuição de propriedades físicas por um artefato é o desenvolvimento de processos baseados em voxel [unidades físicas discretas de material, análogas ao pixel imagético]. Um fluxo de trabalho baseado em voxels permitiria obter propriedades físicas distribuídas gradualmente pela forma do artefato, decorrentes da composição multimaterial planejada. Ainda não há ferramentas digitais para apoiar este tipo de fabricação disponíveis ao público (DOUBROVSKI, et al., 2015). medicina.

Estes avanços previsivelmente alcançariam a indústria de alimentos, a construção civil e a

Aplicações na produção de alimentos e na construção civil vêm sendo experimentadas, 
com variação de matérias-primas (ou ingredientes) e de escala de produto e de máquinas.

Na medicina, para além da impressão de próteses e órteses que já é uma realidade, há também avanços substanciais em pesquisas voltadas para impressão de tecidos vivos e mesmo de órgãos humanos (INSTITUTE FOR REGENERATIVE MEDICINE, 2016), embora as aplicações ainda permaneçam confinadas no contexto da pesquisa acadêmica.

\section{Discussão}

Tendo sidas identificadas tecnologias, caracterizados arranjos produtivos e sistemas de fabricação digital e colocadas em evidências algumas das propriedades emergentes destes sistemas, nesta seção tem início a discussão sobre implicações da difusão destes sistemas para práticas do design.

Historicamente, a simplicidade do artefato é valorizada no campo do design. Esta valorização é compreensível, uma vez que é característica naturalmente esperada de uma boa síntese, qualidade indicativa de soluções elegantes e bem resolvidas.

A simplicidade da forma se alinha bem com interesses produtivos industriais, tipicamente estabelece uma relação direta com a facilidade de produção e, por consequência, com a economia de produção e com o aumento de margens em economias de escala.

A extensão daquilo que é tecnicamente viável, decorrente da adoção de tecnologias de fabricação digital, nos coloca frente a oportunidade de pensar e praticar a complexidade formal e, talvez, ao desafio de redescobrir e reinventar a simplicidade.

As oportunidades abertas pela impressão direta de conjuntos montados - peças consolidadas - reposicionam os sistemas mecânicos enquanto objeto de design, não somente dando acesso a uma grande variedade de componentes eventualmente inacessíveis ou inexistentes, mas também libertando designers dos padrões de componentes industriais.

As perspectivas de produção multimaterial despontam como um fator facilitador e ampliador de possibilidades. $\mathrm{O}$ aumento da variedade de materiais disponíveis previsivelmente colocará as propriedades físicas do objeto no escopo de variáveis das práticas de design.

Embora não seja nenhuma novidade considerar as propriedades de materiais em decisões de design, neste novo contexto elas estariam amplamente disponíveis, permitindo uma liberdade de configuração sem precedentes.

A impressão 3D e outras tecnologias de fabricação digital estão de fato removendo barreiras para a realização de ideias. Da produção de objetos únicos a pequenos lotes, essa abertura de possibilidades é ampliada pelas articulações destas tecnologias em sistemas de fabricação.

Para além do artefato, a virtualização da cadeia de distribuição e do estoque, junto aos sistemas distribuídos de produção, permite testar mercados diretamente, dispensando abordagens tradicionais de pesquisa mercadológica.

As possibilidades decorrentes da liberdade de variação e a customização em massa apontam para o design de sistemas capazes de conduzir a configuração customizada, o desenho de processos ampliados que compreendam, desde a obtenção ou criação da forma, incluindo a possibilidade de participação de usuários, até a entrega do artefato produzido.

De outro lado, a disponibilidade tecnológica e a produção desktop aproximam o designer do processo produtivo e, portanto, das oportunidades e desafios relacionados a estas tecnologias, ao mesmo tempo em que abrem uma janela larga para a experimentação em design. Dessa maneira o design ganha espaço expressivo, relativamente independente da indústria tradicional. 
Das associações entre processos fabris digitais e tradicionais, passando por sistemas ampliados e distribuídos de fabricação digital, há muitos indícios de que é hora de refletir sobre as opções de fabricação e repensar as relações que se desenham na interface entre projeto e produção.

As implicações da adoção da fabricação digital têm longo alcance e amplitude de escopo. Das opções e decisões envolvidas nas práticas criativas até a comercialização de artefatos, passando pela possibilidade de projeto de sistemas fabris não-proprietários e efêmeros para a construção de artefatos com caráter único.

Esperamos que este trabalho auxilie na compreensão deste fenômeno multifacetado e estimule a continuidade das reflexões, necessária ao avanço dos entendimentos sobre o tema.

\section{Referências}

ANDERSON, C. A Nova Revolução Industrial: makers. Rio de Janeiro: Elsevier, 2012.

ANTONELLI, P. States of Design: thinkering. DOMUS, 2011. (http://www.domusweb.it/en/design/2011/07/04/states-of-design-03-thinkering.html). Acesso em: mar. 2018.

BUCHANAN, R. Education and Professional Practice in Design. Design Issues, v. 14, n.2, p. 63-66, 1998.

BÜRDEK, B. E. História, Teoria e Prática do Design de Produtos. São Paulo: Edgard Blücher, 2006.

CLARK, K. B.; WHEELWRIGHT, S. C. Managing New Product and Process Development. New York: Free Press, 1993.

DOUBROVSKI, E. L. et al. Voxel-based Fabrication Through Material Property Mapping: a design method for bitmap printing. Computer-Aided Design, v. 60, p. 3-13, 2015.

FAB FOUNDATION. What qualifies as a Fab Lab?, 2015. (http://www.fabfoundation.org/index.php/what-qualifies-as-a-fab-lab/index.html). Acesso em: mar. 2018.

FORD, E. Getting Started with CNC. San Francisco: O'Reilly - Maker Media, 2016.

GERSHENFELD, N. FAB: the coming revolution on your desktop. New York: Basic Books, 2005.

GERSHENFELD, N. How to Make Almost Anything: the digital fabrication revolution. Foreign Affairs, V.91, N.43, p.43-57, 2012.

GIBSON, I.; ROSEN, D.; STUCKER, B. Additive Manufacturing Technologies: rapid prototyping to direct digital manufacturing. New York: Springer, 2010.

HERTENSTEIN, J. H.; PLATT, M. B.; VERYZER, R. W. What is 'Good Design'?: an investigation of the complexity and structure of design. Design Management Journal, v. 8, n. 1, 2013. p. 8-21.

HOPKINSON, N.; HAGUE, R. J. M.; DICKENS, P. M. (Eds.). Rapid Manufacturing: an industrial revolution for the digital age. Sussex: John Wiley \& Sons, 2006.

INSTITUTE FOR REGENERATIVE MEDICINE. Using 3D Printing Technology to Print Organs and Tissue. Institute for Regenerative Medicine - Wake Forest School of Medicine, 2016. (http://www.wakehealth.edu/Research/WFIRM/Our-Story/Inside-the-Lab/Bioprinting.htm).

Acesso em: mar. 2018. 
LIPSON, H.; KURMAN, M. Fabricated: the new world of 3d printing. Indianapolis: Wiley, 2013.

LÖBACH, B. Design Industrial: bases para a configuração de produtos industriais. São Paulo: Edgard Blücher, 2001.

LOPES, J. et al. (Eds.). Tecnologias 3D: desvendando o passado, modelando o futuro. Rio de Janeiro: Lexikon, 2013.

MAGALHÃES, C. F. D. A Prática Reflexiva no Design Estratégico: fundamentos do design para uma indústria criativa. In: COUTO, R. M. D. S., et al. Formas do Design: por uma metodologia interdisciplinar. 2. ed. Revisada e Ampliada. Rio de Janeiro: Rio Books, 2014. p. 147-163.

OXMAN, N.; KEATING, S.; TSAI, E. Functionally Graded Rapid Prototyping. Innovative Developments in Virtual and Physical Prototyping. Proceedings of The 5th International Conference on Advanced Research and Rapid Prototyping. London: CRC Press, Taylor \& Francis Group. 2012. p. 483-490.

REPRAP. RepRap History. In: RepRap, 2011. (http://reprap.org/wiki/RepRap_history). Acesso em: mar. 2018.

SCHÖN, D. A. The Reflective Practitioner: how professionals think in action. New York: Basic Books, 1983.

SCHRAGE, M. Serious Play: how the world's best companies simulate to innovate. Massachusetts: Harvard Business School Press, 2000.

VOLPATO, N. (Ed.). Prototipagem Rápida: tecnologias e aplicações. São Paulo: Edgard Blücher, 2007. 